\title{
Transformaciones estéticas: la narcocultura, la producción de valores culturales y la validación del fenómeno narco
}

\section{Artículo de reflexión}

\section{Jesús Antonio Pardo León}

Secretaría de Educación del Distrito, Bogotá, Colombia japardol321@hotmail.com

Recibido: 11 de diciembre de 2017

Aprobado: 15 de diciembre de 2017

Cómo citar este artículo: Pardo León, Jesús Antonio (2018). Transformaciones estéticas: la narcocultura, la producción de valores culturales y la validación del fenómeno narco. Calle14: revista de investigación en el campo del arte, 13 (24) pp. 400-409. DOI: https://doi. org/10.14483/21450706.13534 


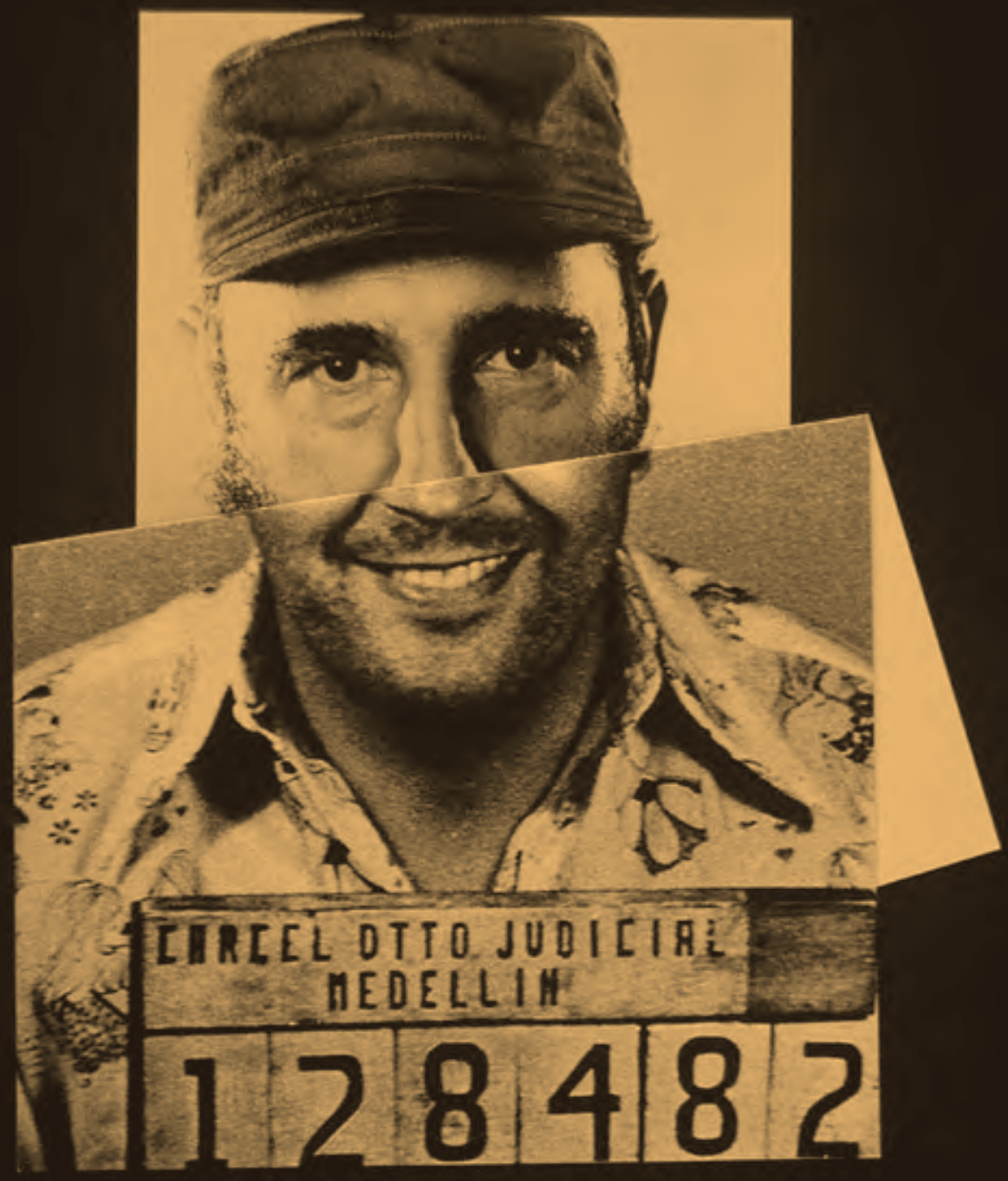


Transformaciones estéticas: la narcocultura, la producción de valores culturales y la validación del fenómeno narco

\begin{abstract}
Resumen
El contexto colombiano de las últimas tres décadas ha generado formas culturales y representaciones sociales emergidas de la crisis e inestabilidad social que ha caracterizado al país durante este período de tiempo. El siguiente trabajo tiene como objetivo realizar una contextualización e interpretación del fenómeno del narcotráfico a nivel local; paralelamente, definiremos herramientas conceptuales para acercarnos a un objeto específico, el caso de la producción de material estético identificado con las nuevas escalas de valor, producto de la cultura generada por el narcotráfico. Por medio de conceptos de tipo sociológico analizaremos estéticamente la configuración e instalación de los nuevos valores "narcos" a través de un estudio del caso Rodrigo D. No Futuro y la producción televisiva alrededor del fenómeno narco.
\end{abstract}

\title{
Palabras claves
}

Narcotráfico, narcocultura, narcoestética, sicaresca.

Aesthetic Transformations: narco-culture, the production of cultural values and the validation of the narco phenomenon

\begin{abstract}
The Colombian context of the last three decades has generated cultural forms and social representations emerged from the crisis and social instability that has characterized the country during this period of time. The following pages aim to contextualize and interpret the phenomenon of drug trafficking at the local level. In parallel, we will define conceptual tools to approach a specific object: the case of the production of aesthetic material identified with a new value scale, product of the culture stemming from drug trafficking. By means of sociological concepts, we will aesthetically analyze the configuration and installation of the new "narco" values through a study of the Rodrigo D. No Futuro case and of the television content produced around the narco phenomenon.
\end{abstract}

\section{Keywords}

Drug trafficking, narco-culture, narco-aesthetics, sicaresca.

Transformations esthétiques : la narcoculture, la production de valeurs culturelles et la validation du phénomène narco.

\section{Résumé}

Le contexte colombien des trois dernières décennies a généré des formes culturelles et des représentations sociales issues de la crise et de l'instabilité sociale qui ont caractérisé le pays pendant cette période. Les pages suivantes ont pour objectif de contextualiser et d'interpréter le phénomène du trafic de drogue au niveau local. Nous définissons des outils conceptuels pour garantir l'approche d'un objet spécifique, dans ce cas, la production de matériel esthétique identifié avec la nouvelle échelle de valeurs, issue de la culture générée par le trafic de drogue. Grâce à des concepts sociologiques, nous analysons esthétiquement la configuration et l'installation de nouvelles valeurs « narco » à travers une étude de Rodrigo D. No Futuro et de la production télévisuelle autour du phénomène narco.

\section{Mots clés}

Trafic de drogue, narcoculture, narco-esthétique, sicaresca. 
Transformações estéticas: narcocultura, produção de valores culturais e validação do fenômeno do narcotráfico

\section{Resumo}

O contexto colombiano das últimas três décadas gerou formas culturais e representações sociais surgidas da crise e da instabilidade social que caracterizaram o país nesse período de tempo. 0 trabalho a seguir tem o objetivo de contextualizar e interpretar o fenômeno do tráfico de drogas no nível local. Paralelamente, definiremos ferramentas conceituais para abordar um objeto específico, o caso da produção de material estético identificado com a nova escala de valores, produto da cultura gerada pelo tráfico de drogas. Por meio de conceitos sociológicos, analisaremos esteticamente a configuração e a instalação dos novos valores "narcos" com um estudo do caso Rodrigo D. No Futuro e da produção televisiva em torno do fenômeno narco.

\section{Palavras chaves}

Tráfico de drogas, narcocultura, narcoestética, sicaresca.

Transformaciones estéticas: la narcocultura, panga pallai, kawachispa, imasam kaugsai, ka kai tarpuspa

\section{Maillallachiska:}

Nukanchipa atun llagta Colombia sutipi kausanchi ajai llakiikunawa, taimi llapa panga suti coca chimanda llukanchi allilla iuraringa y kawanga imasam pudinchi,sug tarpuikuna katichinga chasa mailla mailla tuturingami manakagpi chasallata ñugpasina wañuchiillami kangapkakuna chimanda iuarinsunchi allilla.

\section{Rimangapa Ministidukuna:}

Mana allilla rurai, panga tarpuspa kaugsai, ambi pangata ruraska. 
"Nuestro Dios es el dinero y sin él el hambre está, traficantes se asesinan sin poderlo disfrutar. Lo deseas, lo acaricias y por el la vida das. El sistema lo ha creado y tú lo conservarás." "Dinero". Peste mutantex ${ }^{1}$ (Gaviria, 1988)

\section{Contextualización}

Colombia es el escenario de una compleja red de relaciones de poder económico, político y militar; con una memoria marcada por la violencia, es espectadora de cómo las formas de esta violencia se metamorfosean y cambian de rostro a través del tiempo. Uno de esos episodios puede llamarse el "problema Narco". El narcotráfico emerge con el crecimiento desmedido de los cultivos de coca en el país y el apogeo de los grandes carteles de la producción y comercialización de droga a partir de los años ochenta en adelante. La década de los ochenta se encuentra atravesada por una violencia llamada "del Conflicto Armado" (Palacios y Safford, 2002), definida como una "lucha insurreccional de organizaciones guerrilleras cuyo fin es transformar revolucionariamente el orden social y el Estado que lo protege, y la respuesta de los institutos castrenses y de organizaciones paramilitares" (Palacios y Safford, 2002, p. 643).

Es en dicha década cuando se presentan acontecimientos claves para la historia del conflicto: por una parte, acciones violentas por parte de la izquierda y derecha política en el país, como lo es la toma de la Embajada de la República Dominicana en 1980 y la del Palacio de Justicia en 1985 - por parte de agentes de la insurgencia urbana guerrillera del M-19-, además de otras tomas y actos belicosos por parte de las guerrillas; sumado a esto, el contra-ataque de la derecha y el paramilitarismo por medio del exterminio sistemático de la Unión Patriótica a mediados de los ochenta, aportando al debilitamiento del Partido Comunista, que ya está golpeado por la disolución de la URSS y el fin de la Guerra Fría (lo cual lleva a las FARC a independizarse del PC, postulando una nueva perspectiva militar y política propia a finales de la década), agudizan la violencia y el terror en el país (Palacios y Safford, 2002).

Por otra parte, los ochentas ven el comienzo de una historia de procesos de paz, partiendo de Belisario Betancur (1982-1986) -los diálogos de La Uribe (Meta)

1 Banda sonora de la película "Rodrigo D. No futuro" del director Víctor Gaviria. (Medellín, 1988). con las FARC, de Corinto (Cauca) y el Hobo (Huila) con el M-19 y el EPL en 1984- y Virgilio Barco (1986-1990) logrando la desmovilización del M-19 y el EPL en 1990-, que continuaron con presidentes posteriores y aún continúan con el actual proceso de paz adelantado por el presidente Juan Manuel Santos y la guerrilla de las FARC.

Pero el elemento más relevante para este documento dado en el tiempo descrito está marcado por el narcotráfico, el cual encontró en zonas de influencia de la guerrilla de las FARC (como el Magdalena Medio, Urabá, Meta, Caquetá, Putumayo y Guaviare) un espacio propicio para el cultivo y procesamiento de los alcaloides; logra alianzas de diversas formas y compromisos con los guerrilleros en un principio, pero termina rompiéndolas a finales de la década (Palacios y Safford, 2002).

El narcotráfico cambiará las condiciones del poder en todos los niveles; económico, político, social y cultural. A finales de los ochenta, al romper con la guerrilla, los narcos establecen pactos con las hegemonías políticas de la nación, donde se permite e invisibiliza el llamado "traqueteo" a cambio de liquidar la oposición en la esfera de la política (utilizando el sicariato para tal fin), y fortaleciendo el paramilitarismo. En los años noventa la guerra en Colombia se agudiza; el fin de la "Guerra Fría" impone el modelo Neoliberal a escala global, trayendo al país relaciones comerciales desventajosas para los productores nacionales e impulsando las políticas de privatización de las empresas públicas. En los grandes centros de consumo del país, sus principales ciudades, los efectos de la "apertura económica" promovida por el presidente Gaviria son amortiguados por el narcotráfico en un principio; pero la crisis profunda se vive en el campo, donde gran parte de la tierra cultivable está en manos de productores de coca y amapola, provocando la marginación del campesinado y el fenómeno del desplazamiento (Rueda Fajardo, 2009).

Los noventas son testigos de las transformaciones económicas y sociales que propician que la empresa del narcotráfico prospere. La marginación de la población campesina acelera la producción de opio y cocaína en lugares como el Cauca y el Tolima, propiciando una guerra a gran escala en los actores del conflicto (narcos, "paras" y guerrilla) interesados en el control territorial y, en cierto modo, el monopolio de la producción y comercialización de sustancias psicoactivas.

La zona antioqueña es uno de los lugares donde el impacto del narcotráfico transforma las escalas de valores, el terreno psicológico-social y las formas 
culturales con gran intensidad; la pobreza, el desempleo, la marginalidad y la violencia, son el entorno donde la organización del narcotráfico evoluciona, y, en lugares como Medellín, “los jóvenes pobres encontraron nuevas oportunidades empleándose como sicarios en un contexto de banalización de la muerte" (Palacios y Safford, 2002, p. 654).

Es durante la década de los noventas que el problema del narcotráfico es denunciado y trabajado en los círculos artísticos e intelectuales. A principios de los noventa aparece la narrativa Sicaresca (término acuñado por Hector Abad Faciolince en 1994), con ejemplos como la película del director Víctor Gaviria "Rodrigo D. No Futuro", y las novelas "Rosario Tijeras" del escritor Jorge Franco Ramos y "La Virgen de los Sicarios" escrita por Fernando Vallejo; ambas llevadas al cine comercial entre los años noventa y el principio del segundo milenio. En el terreno de las artes plásticas, durante la segunda mitad de la década, la posición crítica frente al fenómeno del narcotráfico se hará presente claramente; ejemplo de esto serán exposiciones como "Scars=Pleasure+Sacrifice" de 1995, la "V Bienal de Arte de Bogotá" de 1996, y la exposición "Status Quo" de 19992; además de obras plásticas como "Cajas Fucsia con Estrellas Brillantes" de Juan Fernando Herrán y otros artistas relevantes de este periodo como lo son Fernando Arias, José Alejandro Restrepo y Miguel Ángel Rojas (Rueda Fajardo, 2009) y (Rueda Fajardo, 2009a).

Las últimas tres décadas hemos sido testigos de la aparición de las concepciones del "Narco" y de "narcotráfico", y también de su popularización y sofisticación. Luego de la desarticulación de los carteles de Medellín y Cali, el negocio del narcotráfico sufre cambios importantes, ya que desaparecen las grandes infraestructuras ${ }^{3}$ que componían un negocio multimillonario, dirigido por una cabeza visible que ostenta el poder: el capo. Entrados los años noventa y en adelante, los cultivos de coca y amapola aumentaron considerablemente, pero el dinero del narcotráfico no se hace visible en el país, las inversiones del narcotráfico se dirigen a países donde se pueda gozar de "paraísos fiscales" como el Ecuador, Estados Unidos y Suiza. El proyecto del narcotráfico, aunque ilegal, se va especializando en la configuración de redes de cultivo, producción y tráfico, que cada vez se van conformando

2 "Status Quo" gozó de gran importancia, pues en palabras de Rueda "anuncia para las artes visuales colombianas su ingreso en el género narco". (Rueda Fajardo, Musa Hibrida, 2009).

3 El cartel de Medellín alcanzó a tener una organización de alrededor de 3.000 personas vinculadas. (Revista Semana, 2000).

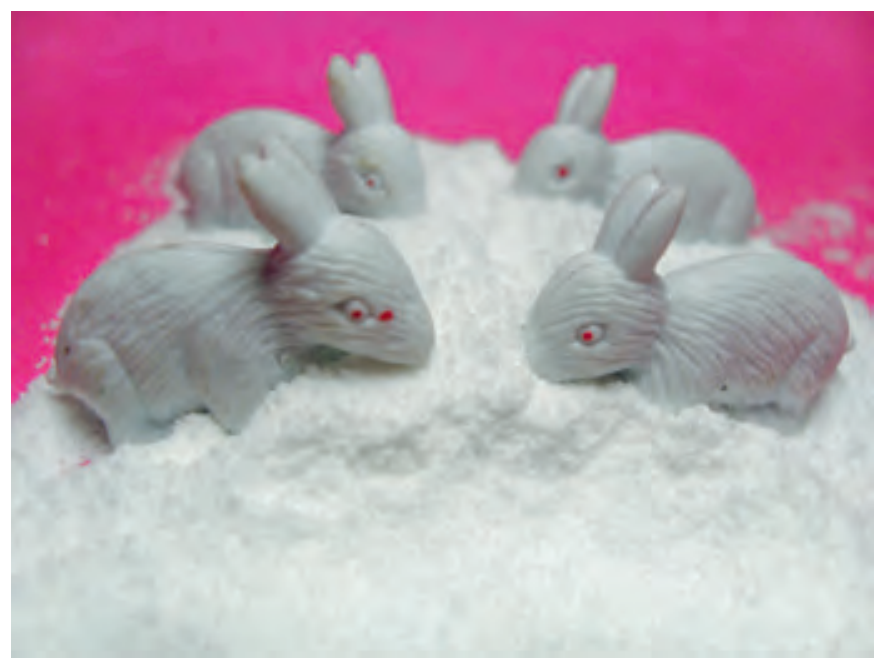

Fotografía: Edinson Quiñones, (2015). Feliz Año Nuevo. Cortesía del artista.

con más aceptación y legitimación del fenómeno por parte de la sociedad contemporánea global.

El desarrollo de la cultura producida por el fenómeno del narcotráfico se naturaliza y populariza con mayor profundidad al ser llevada a las grandes masas por medio del cine y la televisión, aunque ya se había expandido en los campos intelectual, artístico, literario y periodístico terminando los años ochenta. "las representaciones sobre el narcotráfico recreadas en los medios de comunicación no sólo sirven para darle sentido a una serie de elementos que la gente conoce o intuye, sino que participan en la producción de prácticas cotidianas desde las cuales la gente aprende a vivir con ese mundo" (Ovalle, 2010, p. 10). La noción de "Narco" se va configurando como una construcción social aceptada, clasificada entre las ocupaciones económicamente más rentables, configurada por grandes redes internacionales de tráfico y producción y amparada por los valores económicos y éticos del neoliberalismo (Ovalle, 2010). "Aunque los cálculos sobre la magnitud económica del negocio del narcotráfico son imprecisos, no cabe duda de que ha generado nuevos comportamientos y códigos de valores (el dinero fácil), unidos a los viejos (el honor machista, o que «la vida no vale nada»)" (Palacios y Safford, 2002, p. 653).

\section{Definición de los conceptos para el análisis estético}

Siguiendo el texto de Henao (2010) podemos encontrar una conexión entre el fenómeno narco y la psiquis social por medio del concepto de Representación 


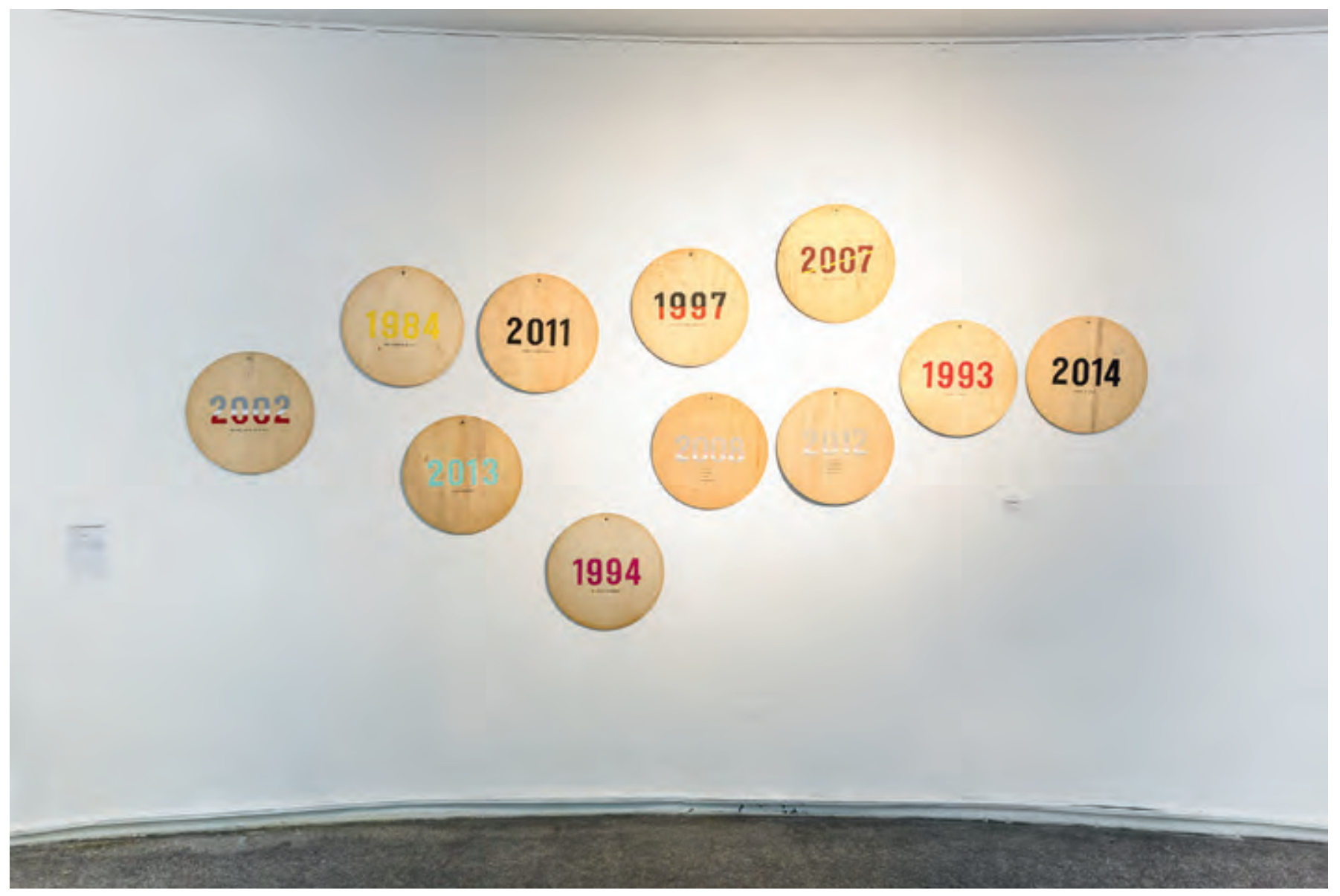

Imagen 1. Exposición Una linea de polvo: arte y drogas en América Latina. Museo de Arte Contemporáneo, (Bogotá, 10 de noviembre de 2017). Curador: Santiago Rueda. Cortesía: curador.

Social. Este concepto se interpreta como un sistema de valores, creencias y costumbres que fundamentan y justifican prácticas de individuos en determinados grupos sociales; su función está en presentarse como una ética que establece ideales para direccionar a los sujetos por medio de la identificación con propósitos sociales y la noción de "realización personal" o "iniciación", además de proporcionar sistemas significativos que facilitan las conexiones y comunicaciones entre un grupo, sus modos de ver el mundo y dar valor y sentido a los objetos de ese mundo.

Según la teoría de la representación Social, las RS emergen bajo un factor denominador histórico-social que condiciona su formación y la configuración de los sistemas de significación que las conforman; dicho factor está marcado por la crisis y los conflictos sociales. Las RS son la forma de explicación y entendimiento de acontecimientos complejos y traumáticos; de esta manera justifican su manera de ser y de actuar diferenciando unos grupos sociales de otros y, finalmente, naturalizan las formas de violencia producto de las tensiones resultantes de la diferenciación social (Henao Henao, 2010).

El fenómeno narco es candidato a ser una de las representaciones sociales más intensas de las últimas décadas. Dentro del discurso de investigadores sobre el tema se han ido develando los sistemas de valores e interpretando las condiciones histórico-sociales $y$, partiendo de ello, se han concebido definiciones propias para poder acceder a la información y poderla organizar y pensar; nociones tales como las dadas por Omar Rincón (narco.cultura y narco.estética), Margarita Jácome (la sicaresca, a través de Héctor Abad F.) y Santiago Rueda (narcochic), entre otros.

Toda esta cultura nacida del narcotráfico ha sido definida por Rincón (2009) como narco.cultura, que da legitimación a la violencia y promociona valores capitalistas tales como el consumismo y el derroche de energía e insumos, por ejemplo. Permea la sociedad en general generando unos estereotipos puntuales y unos gustos generalizados dentro de grupos familiarizados con el 
fenómeno narco, unos más populares que otros, más sofisticados, o más conservadores. A partir de lo anterior se puede argumentar que la influencia del narcotráfico afectó a todas las capas sociales de la población colombiana, sin medir profundamente el influjo internacional de este, como la mistificación del consumo de drogas en EE.UU y Europa en el cine, la moda, la música y la publicidad.

A los modelos estéticos populares Rincón los llama narco.estética, caracterizada por "ostentosa, exagerada, desproporcionada y cargada con símbolos que buscan dar status y legitimar la violencia" (Cobo, 2008). Según Rincón (2009), la narco.estética está caracterizada por varios estilos o gustos marcados por la narco. cultura: el estilo norteamericano capitalista del nuevo rico o el rico colombiano de las montañas de Antioquia, como el estilo mexicano del mafioso narcotraficante al modo "norteño".

Dentro de esta narco.cultura encontramos otros relatos como lo es la sicaresca y el narcochic. La sicaresca está dada por la fascinación por los sicarios, la truculencia y la pasión por los excesos. El sicario es el joven producido por la cultura de la violencia, de la muerte y de la necesidad, es el huérfano de la narco.cultura; es un héroe efímero y marginal marcado por la vida rápida, el gusto por el exceso y la naturalización de la muerte (Rincón, 2009).

Esta literatura del sicario aparece en el escenario de la crisis social colombiana dada por su inestabilidad política, las influencias externas neoliberales e internas de las fuerzas ilegales, y la reducción de los espacios de participación y de opinión frente al control de los grandes medios de comunicación por parte de algunos sectores económicos y políticos. La crítica de los intelectuales y artistas frente a la problemática de lo narco empieza a desarrollarse en la sicaresca.

Lo narcochic es un concepto que hace referencia a una exposición titulada "Narcochic - Narcochoc" realizada en Francia en el año 2003, la cual tenía por finalidad enseñar la estética popular —o folclor- asociada a la cultura del narcotráfico "en sus aspectos más pintorescos" (Rueda Fajardo, Narcochic, 2009), encontrando manifestaciones no solamente colombianas sino también de impronta mexicana en la formación de lo narcochic. El heroin chic (Rueda Fajardo, 2009) surgido en Europa y EE.UU. podría enumerarse dentro de esta cultura narcótica transnacional. Lo narcochic son los valores estéticos y éticos establecidos y legitimados por el establecimiento comercial global; la popularización a niveles masivos de la cultura narco.

\section{Análisis de Caso}

Hay muchos documentos relacionados con la representación social del fenómeno narco, basta con observar bases de datos como las de la página web Mama Coca para encontrarse con casi 500 títulos desde finales de los años setenta hasta el año 2015 (sin contar la literatura sobre las experiencias con las sustancias psicoactivas anteriores a la fecha) (MamaCoca, 2011). Tomaremos referencia del cine y la televisión realizadas bajo los valores de la narco.cultura para reflexionar sobre cómo la sicaresca —identificada por su posición crítica y de denuncia frente al fenómeno narco-, dio paso a la estética narcochic, estilo legitimado y aceptado desde los grandes medios de comunicación y el comercio global.

Una de las películas más importantes dentro de la temática de la narco.cultura es el filme de Víctor Gaviria titulado "Rodrigo D. No Futuro", se trata de la obra cinematográfica representante del género de la Sicaresca por excelencia. La historia relata los acontecimientos ocurridos en 1988 en Medellín (Colombia) a un joven que no cumple aún los 20 años de edad y muestra un fuerte desinterés por la vida luego de la muerte de su madre, lo único que interesa al muchacho es la música y la cultura punk; tras frustrados intentos por adquirir una batería, consigue unas baquetas, que lo acompañarán en el transcurso del film. Su entorno social son las comunas de Medellín, donde la crisis social es evidente, los jóvenes contemporáneos a Rodrigo (protagonista de la película) se encuentran inmiscuidos en negocios al margen de la ley como el hurto, el tráfico de drogas y el sicariato; por lo cual varios de ellos son aniquilados. Luego de visitar a una amiga de su madre y ver fotos juntos, Rodrigo sube al piso 20 de un edificio de la ciudad y salta al vacío mientras suena la canción de la banda Mutantex "No te Desanimes, Mátate". Al mismo tiempo, es asesinado uno de los jóvenes vecinos de Rodrigo -Ramón-, por conflictos con jóvenes del mismo contexto social.

El termino Sicaresca inicialmente fue acuñado por Héctor Abad Faciolince en el año de 1995 en un artículo en la revista Semana. Termino que adopta y profundiza Margarita Jácome en su libro sobre la novela sicaresca. La calificación de sicaresca que se le otorga al largometraje de Gaviria es dado por Rueda en su ensayo "Una Tumba en Colombia: Los Años Ochenta". Las 


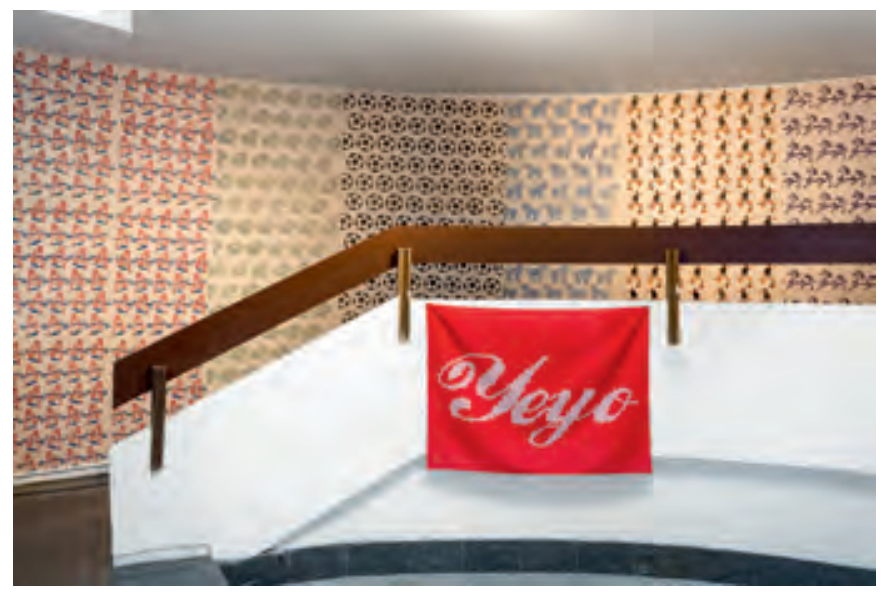

Imagen 2. Exposición Una linea de polvo: arte y drogas en América Latina. Museo de Arte Contemporáneo, (Bogotá, 10 de noviembre de 2017). Curador: Santiago Rueda. Cortesía: curador.

características del género sicaresco, en Jácome, están atravesadas por "una realidad tan compleja y caótica como la situación política, económica y social colombiana de las dos últimas décadas del siglo XX" (Jácome, 2009, p. 15); además de tener como componente fundamental la legitimación y naturalización de la violencia, el género articula "temas existenciales" como "el amor, el desengaño, los viajes y la separación", exponiendo "la caída de los valores tradicionales" y "los cambios culturales de las últimas décadas en Colombia" (Jácome, 2009, pp. 15-16).

Por su parte, Rueda habla de "Rodrigo D. No Futuro" como representante de la sicaresca junto a novelas llevadas al cine en la década de los noventas y principio del segundo milenio como La Virgen de los Sicarios y Rosario Tijeras:

“No Futuro es una máxima del punk en todo el mundo. Indica la amenaza de la guerra nuclear, pero sobre todo el abandono que en la sociedad postindustrial se tiene para todo aquello que no sea la imagen de un producto consumible, devorable. No Futuro es la máxima de lo que se ha llamado postmodernismo, el mundo de la publicidad en donde todo se ha reducido a un inmenso basurero. El tiempo se ha detenido en un presente comestible, en la inminencia del consumo. El presente en que vive el producto encerrado en su empaque al vacío, que de un momento a otro será comido, consumido y luego será basura en el basurero de todas las cosas" (Rueda Fajardo, 2009c).

En el año 2006 se estrena en televisión la serie "Sin tetas no hay paraíso", de Gustavo Bolívar, alcanzando uno de los rating más altos en la televisión colombiana y gran reconocimiento internacional (con una exitosa audiencia en toda Suramérica y Centroamérica y adaptada por la televisión española y norteamericana); los temas específicos de la novela llevada a la televisión son el narcotráfico y la figura del sicario. "Así, la estética que pobló buena parte de los productos culturales de cierto nivel en los años 90, hoy, casi 15 años después, se convierte en un producto televisivo sobreexplotado: La viuda de la mafia, El cartel de los sapos, Inversiones el $A B C$, Sin tetas no hay paraíso (en formato de telenovela), entre otras, mandan la parada. Tanto es así, que desde que no hay sicarios en la televisión, los ratings de las telenovelas de RCN se desplomaron" (2009).

El redactor del artículo citado arriba de la revista "Semana" titulado "De la sicaresca a la narcoestética", interpreta y enfrenta el gusto generalizado y popularizado por la narcocultura (la narcoestética) con un género novelesco de carácter crítico y profundidad estructural como la sicaresca. Mientras que esta (la sicaresca) tiene una visión realista y humana sobre la juventud sumida en la narcocultura, en el sinsentido social, en la desesperanza y la violencia absurda, la narcoestética de los grandes medios de comunicación, no proponen una denuncia frente al público de unas prácticas e imaginarios de la cultura del narcotráfico, sino que su preocupación es satisfacer una demanda de nuevos valores sociales y vender un nuevo estilo que ha ido naturalizándose a través del tiempo.

Ahora, la distinción entre narcoestética y narcochic se hace confusa. El gusto por lo ostentoso, exagerado, con miras a alcanzar un reconocimiento social y legitimar las formas de violencia mafiosa orientada por los modos del millonario norteamericano, colombiano o mexicano, es lo registrado por Rincón como narco.estética; lo narcochic es la tendencia a aceptar y naturalizar la narcoestética, abandonando el juicio sobre el "mal gusto" de lo narco, sobre un estilo chic de carácter transnacional identificado y producido por la narcocultura a nivel global; lo narcochic es la transformación de la escala de valores respecto al "buen" o "mal" gusto estético a partir de la legitimación internacional de la narcocultura.

La pregunta que queda es ¿Cuáles son las condiciones para que la narcoestética se convierta en chic? Para intentar responder esa pregunta tomaremos la concepción de Anomia Social de Carlos Rincón (2014). La Anomia Social está emparentada con las nociones de "desarrollo" y "subdesarrollo", utilizadas por el presidente Truman en 1949 para determinar el ideal de 
nación y vender al mundo los valores de la sociedad industrial capitalista; pero el concepto de "desarrollo" tomado en este sentido, no diferencia entre el desarrollo económico de un país y su desarrollo y progreso social (fácilmente entendible, ya que la finalidad del capitalismo no es la sociedad sino la acumulación de capital).

La "Bonanza marimbera" de finales de los años setentas y el gran auge del narcotráfico a partir de los años ochenta en adelante en Colombia trae para el país el enriquecimiento rápido y sin control de grupos al margen de la ley $y$, dentro de ella, la abrupta influencia de un sector del mercado (el narcotráfico) que no era conocido en el pasado pero que trajo consigo un progreso económico que no iba de la mano con el progreso social ni con las escalas de valores tradicionales. "la anomia sugiere que solo quebrantando, con un despliegue de energías criminales y violencia, las reglas que hasta ese momento habían contribuido al mantenimiento del sistema vigente, es posible realizar expectativas legítimas: alcanzar movilidad social y mejoramiento económico" (Rincón, 2014, p. 52).

La inestabilidad política y económica de la nación, junto al fenómeno generalizado del narcotráfico, generaron unas formas de representaciones sociales acordes con su momento histórico; la aceptación e identificación con los valores del "todo vale", del "dinero fácil", del derroche y de la superficialidad del ornamento y de la estética corporal femenina, identificados con el "mal" gusto narcoestético, se convierten en narcochic e imponen las nuevas formas y direcciones que debe tener el gusto popular, pero sobre todo, la adopción de un estilo de vida caracterizado por la narcocultura como estándar de valores estéticos y éticos.

\section{Referencias}

Abad Faciolince, H. (10 de julio de 1994). Lo Último De La Sicaresca Antioqueña. El Tiempo.

Cobo, A. (28 de junio de 2008). La Estética del Narcotráfico. Esfera Pública. Obtenido de http:// esferapublica.org/nfblog/la-estetica-del-narcotrafico/ De la Sicaresca a la Narcoestética. (13 de junio de 2009). Revista Semana. Obtenido de http://www.semana.com/cultura/articulo/ de-sicaresca-narcoestetica/104078-3
Gaviria, V. (Dirección). (1988). Rodrigo D. No Futuro. [Película].

Henao Henao, S. (2010). Representaciones Sociales del Consumo de "Drogas" y de las Intervenciones Respectivas en un Contexto Local: la Universidad de Antioquia en Medellín, Colombia. Editorial de la Universidad de Granada.

Jácome, M. (2009). La Novela Sicaresca. Testimonio, Sensacionalismo y Ficción. Medellín: Fondo Editorial EAFIT.

MamaCoca. (2011). wp.mamacoca.org. Obtenido de http://www.mamacoca.org/docs_de_base/ La_Representacion_Social_del_narcotrafico/ Representacion_Social_Narcotrafico_en_el_Imaginario_ Popula_2011.html

Ovalle, L. P. (enero-junio de 2010). Construcción Social del Narcotráfico Como Ocupación. Revista CS de la Facultad de Derecho y Ciencias Sociales de la Universidad Autonoma de Baja California Icesi(5), 92-122. doi:http://dx.doi.org/10.18046/recs.i5.453

Palacios, M., y Safford, F. (2002). Colombia: Pais Fragmentado, Sociedad Dividida: Su Historia. Bogotá: Editorial Norma.

Revista Semana. (5 de junio de 2000). Los Nuevos Narcos. Revista Semana.

Rincón, C. (2014). De Cómo los Colombianos Llegaron a Ser Normativamente Pluriculturales. En C. Rincón, Iconos y Mitos Culturales En la Invención de la Nación Colombiana. Pontificia Universidad Javeriana.

Rincón, O. (Julio-agosto de 2009). Narco.estética y Narco.cultura en Narco.lombia. Revista Nueva Sociedad(22), pp. 147-163.

Rueda Fajardo, S. (2009). Una Línea De Polvo: Arte y Drogas En Colombia. Bogotá: Alcladía Mayor De Bogotá. 